\title{
Clinical outcomes of distal vaginal and vulvar cancer treated with image-guided brachytherapy
}

\author{
Alexander Yaney, MD!, Erin Healy, MD', Xueliang Pan, PhD², Douglas Martin, MD', Allison Quick, MD' \\ 'Department of Radiation Oncology, The Ohio State University Wexner Medical Center, Columbus, OH, USA, ${ }^{2}$ Department of Biomedical \\ Informatics, The Ohio State University Wexner Medical Center, Columbus, OH, USA
}

\begin{abstract}
Purpose: To evaluate treatment outcomes with image-guided brachytherapy (IGBT) for distal vaginal and vulvar cancers.

Material and methods: Women treated for distal vaginal or vulvar malignancies utilizing IGBT were retrospectively reviewed, and acute and late toxicities were retrospectively graded. Descriptive statistical analysis was performed.

Results: Eighteen patients were included, out of which, twelve patients $(66.7 \%)$ were with primary disease of the distal vagina and vulva, most commonly squamous cell carcinoma of the vulva $(n=8,66.7 \%)$, and six with recurrent disease, most commonly recurrent endometrial carcinoma $(n=5,83.3 \%)$. All patients received external beam radiation (EBRT) to a median dose of 45 Gy in 25 fractions, followed by IGBT (range of 15 to 27.5 Gy in 3 to 5 fractions). Mean follow-up was 20.6 months. Mean dose to high-risk clinical target volume (HR-CTV) $D_{90}$ was 72.4 Gy. Mean $D_{2 c c}$ for the rectum, bladder, and urethra were $50 \mathrm{~Gy}, 50.6 \mathrm{~Gy}$, and $62.9 \mathrm{~Gy}$, respectively. Five patients (27.8\%) recurred. Three patients $(16.7 \%)$ had local recurrence, 1 patient $(5.6 \%)$ had distant recurrence only, and 1 patient $(5.6 \%)$ had simultaneous regional and distant recurrence. Grade 3 acute toxicities included $1(5.6 \%)$ vaginal stenosis, $6(33.3 \%)$ dermatitis/ mucositis, $2(11.1 \%)$ vaginal pain, and 1 (5.6\%) vaginal/vulvar infection. Grade 3 late toxicities comprised 3 (17.7\%) cases of vaginal pain and $1(5.9 \%)$ skin/vaginal necrosis. There were no grade 4 or higher toxicities.

Conclusions: Definitive radiation therapy in the form of EBRT and IGBT provides meaningful loco-regional control in women with distal vaginal and vulvar cancers, with mainly skin and vaginal toxicity.

J Contemp Brachytherapy 2021; 13, 4: 419-425 DOI: https://doi.org/l0.5114/jcb.2021.108596
\end{abstract}

Key words: distal vaginal cancer, vulvar cancer, brachytherapy, image-guided, toxicity.

\section{Purpose}

Vaginal and vulvar cancers, whether primary or secondary in nature, are uncommon malignancies [1-5]. Due to its rarity, the treatment of vaginal cancer has been largely modeled on the treatment of cervical cancer [6]. As such, both definitive and palliative radiation in the form of brachytherapy with or without external beam radiation, can play an important role in the treatment of vaginal cancer. These same modalities have proven to be effective in the treatment of vulvar cancer, particularly in patients who are deemed poor surgical candidates [2, 7-9].

The use of image-guided brachytherapy (IGBT) in the treatment of cervical cancer has improved local control of disease, and has decreased both early and late toxicities through better clinical target volume (CTV) and organ at risk (OAR) delineation, while also allowing for dose escalation $[3,6,10]$. Retrospective studies suggest the same benefits may be true for the treatment of vaginal and vulvar cancer $[8,9,11,12]$. While there have been studies examining the efficacy and toxicity of brachyther- apy in the treatment of vaginal and vulvar cancer, no study has focused on outcomes associated with the use of brachytherapy particularly for distal vaginal and vulvar malignancies.

The primary aim of this study was to examine the short- and long-term toxicities as well as outcomes associated with the use of brachytherapy for patients with distal vaginal and vulvar cancer, in the modern era of image guidance.

\section{Material and methods}

All patients treated with IGBT for either primary or secondary distal vaginal, defined as tumors originating and confined to the distal third of the vagina, or vulvar malignancies between 2012 and 2019 were retrospectively reviewed. Data collected included patient demographics, pathology, radiation treatment, chemotherapy details, and toxicity. This study received an institutional review board (IRB) approval. 


\section{Radiation}

All patients were treated with a combination of external beam radiation (EBRT) and IGBT. External beam radiation was given first to the primary disease and grossly involved lymph nodes, based on clinical exam and imaging and nodal volumes at risk. EBRT was then followed by IGBT, which was delivered in 3-5 fractions, based on tumor location and size at the time of brachytherapy to achieve adequate dose to the CTV, as defined below. Syed applicators were the preferred applicators for brachytherapy, but other applicators were used if clinically appropriate. Other applicators utilized included a Capri applicator and a custom multichannel applicator. All patients who were treated with a Syed applicator were treated with a single implant, and received their first brachytherapy fraction on the day of implant placement. They were then treated twice daily, until treatment completion. Patients treated with the Capri applicator or custom applicator were treated on non-consecutive days. Computed tomography (CT) alone was obtained to verify applicator placement and for 3D-based treatment planning prior to 2015. After 2015, magnetic resonance imaging (MRI) pelvis following applicator placement was routinely used in addition to CT for brachytherapy treatment planning to maximize CTV and OAR delin- eation [13]. After these images were obtained, CT and MRI images were fused, if applicable, for contouring for CTV and OARs. CTV for brachytherapy was generated based on the initial extent of disease on pre-treatment imaging, if available, and physical exam while taking into account response to EBRT, as noted on imaging utilized for IGBT and physical exam at that time. OARs contoured included the bladder, urethra, and rectum, as appropriate. Treatment planning was performed in BrachyVision (Varian Medical Systems, Palo Alto, California, USA) to manually optimize CTV coverage while obtaining acceptable OAR dose. Figures 1 and 2 show representative axial images of contouring and planning for brachytherapy for two separate patients. Treatment was delivered via VariSource iridium-192 high-dose-rate (HDR) remote afterloader (Varian Medical Systems, Palo Alto, California, USA).

The goal was to achieve an $\mathrm{EQD}_{2}$ for $\mathrm{CTV} \mathrm{D}_{90}$ of 70-75 Gy while minimizing the dose to surrounding OARs. Goals for OARs were to achieve a total $\mathrm{EQD}_{2}$ dose to $2 \mathrm{cc}\left(\mathrm{D}_{2 \mathrm{cc}}\right)$ to the rectum of less than $75 \mathrm{~Gy}$ and less than $90 \mathrm{~Gy}$ for the bladder [5]. The goal for the urethra for $\mathrm{EQD}_{2}$ dose to $0.1 \mathrm{cc}\left(\mathrm{D}_{0.1 \mathrm{cc}}\right)$ was less than $85 \mathrm{~Gy}$ [14]. The vagina itself was not used as an avoidance structure or OAR for treatment planning.

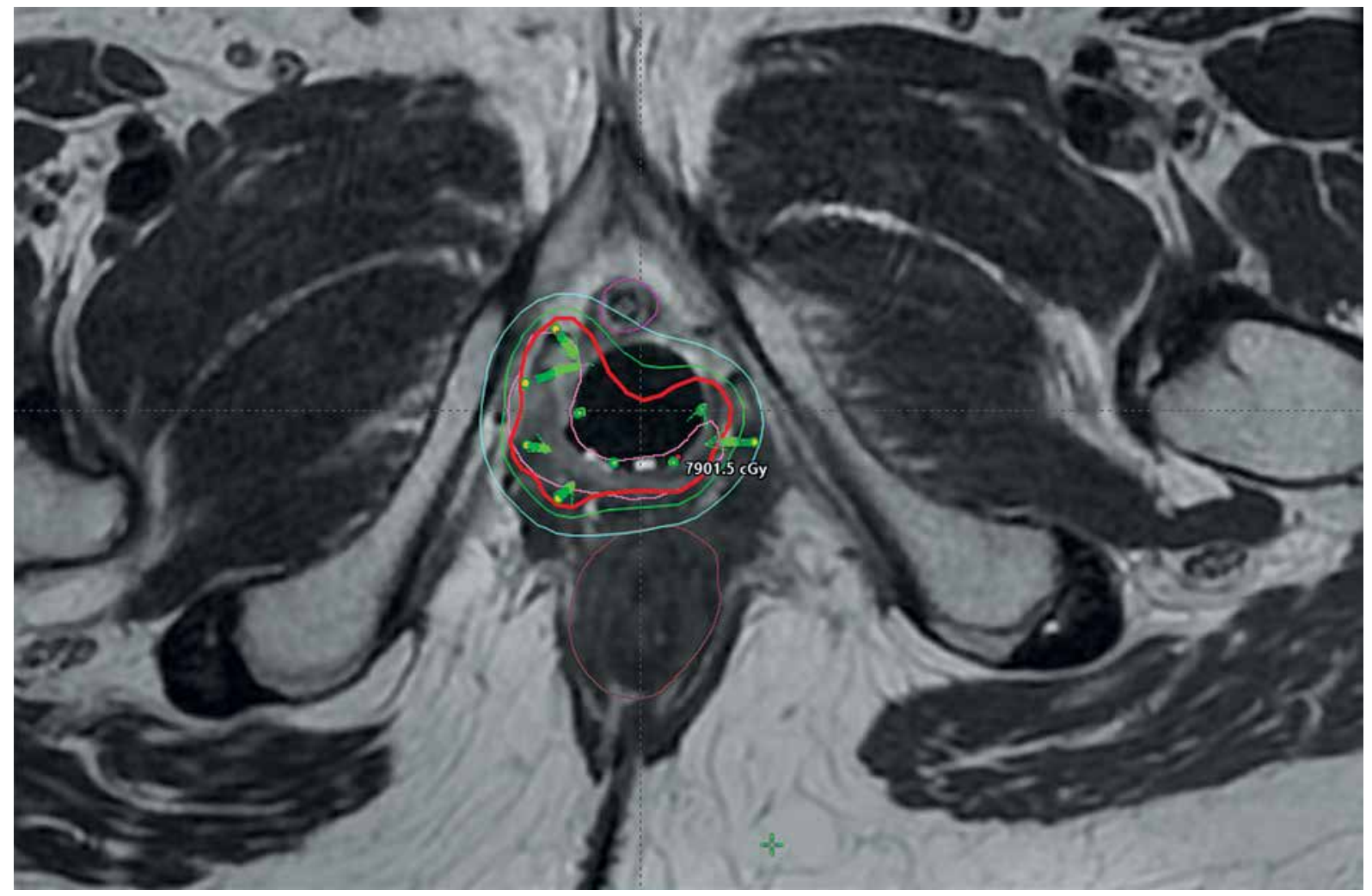

Fig. 1. T2-weighted axial MRI at the time of brachytherapy for a 55-year-old female with a primary FIGO stage II squamous cell carcinoma of the vagina. On physical exam, a vaginal lesion was noted in the distal posterior and lateral walls, right worse than left, extending 2-3 cm into the vagina, with no palpable disease under the urethra or in the anterior vagina. Prior to brachytherapy, she completed $45 \mathrm{~Gy}$ in 25 fractions to the pelvis. Brachytherapy was performed with a Syed applicator. The prescription dose for brachytherapy was $25 \mathrm{~Gy}$ in 5 fractions. $\mathrm{EQD}_{2}$ were as follows: high-risk CTV (HR-CTV) = 74.7 Gy, bladder $=51.2 \mathrm{~Gy}$, rectum $=64.0 \mathrm{~Gy}$, sigmoid $=45.4 \mathrm{~Gy}$, and urethra $=77 \mathrm{~Gy}$. The patient is 21 months after completion of brachytherapy and is currently without evidence of disease. Red $=100 \%$ isodose line, pink $=$ HR-CTV, brown $=$ rectum, magenta $=$ urethra 


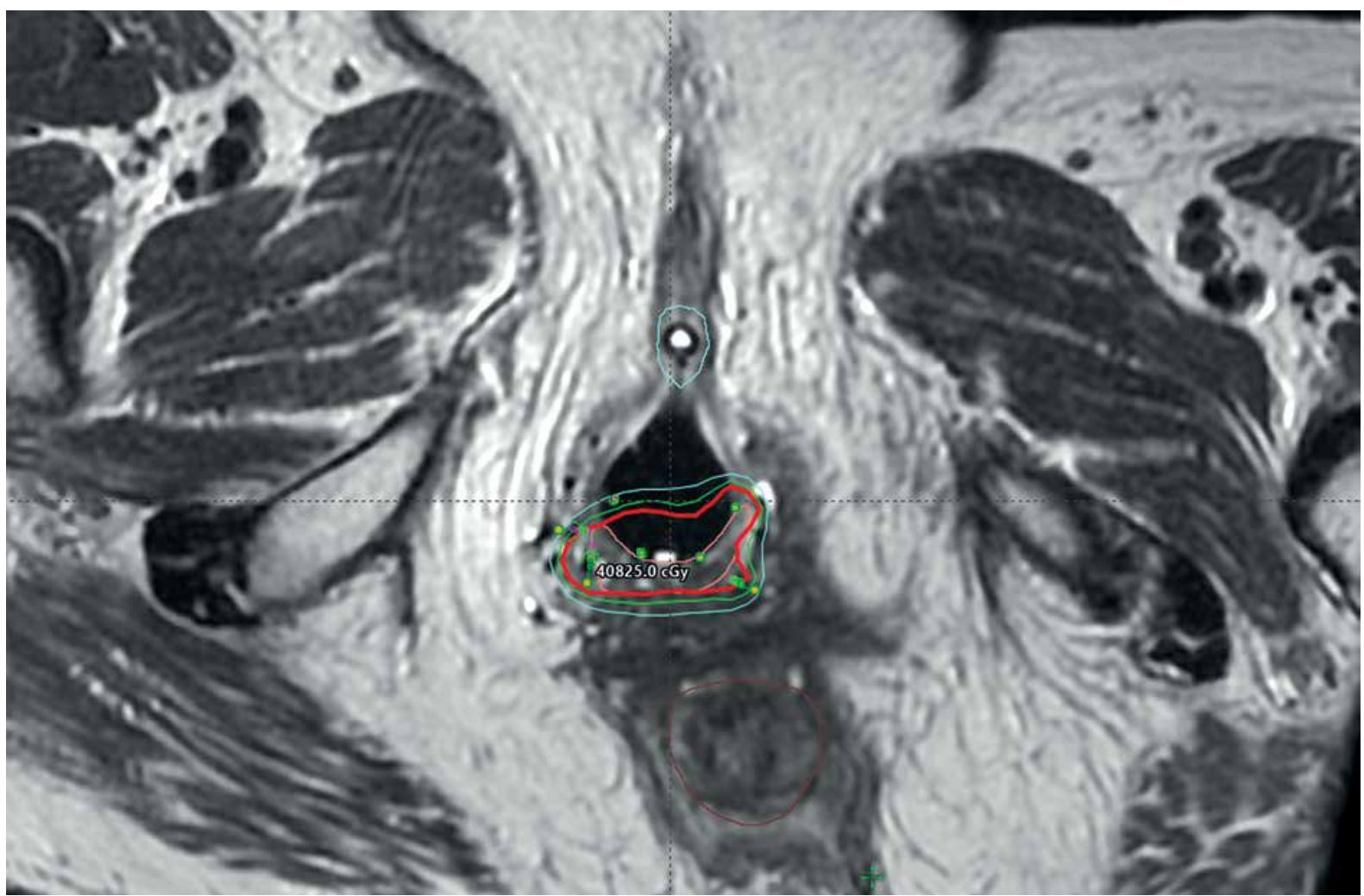

Fig. 2. T2-weighted axial MRI at the time of brachytherapy for a 64-year-old female with history of stage II endometrioid adenocarcinoma s/p TAH-BSO, external beam radiation (EBRT) to $50.4 \mathrm{~Gy}$, and vaginal brachytherapy boost of $6 \mathrm{~Gy}$, who presented with a recurrent disease. On physical exam, she was noted to have a palpable mass in the distal vagina at the introitus extending $2-3 \mathrm{~cm}$ into the vagina, with palpable fullness along the vaginal walls, left greater than right, with no palpable disease under the urethra or in the anterior vagina. She completed a course of EBRT to $30.6 \mathrm{~Gy}$ in 17 fractions prior to brachytherapy, which was performed with a Syed applicator. The prescription dose for brachytherapy was $27.5 \mathrm{~Gy}$ in 5 fractions. EQD 2 were as follows: high-risk CTV $($ HR-CTV $)=70.1 \mathrm{~Gy}$, bladder $=29.4 \mathrm{~Gy}$, rectum $=33.9 \mathrm{~Gy}$, sigmoid $=29.4 \mathrm{~Gy}$, and urethra $=35.5 \mathrm{~Gy}$. The patient is 22 months after completion of brachytherapy and is currently without evidence of disease. Red $=100 \%$ isodose line, pink $=\mathrm{HR}-\mathrm{CTV}$, brown $=$ rectum, cyan $=$ urethra

\section{Toxicity}

Acute and late toxicity outcomes were obtained from review of the patients' records. If not given a grade by the treating radiation oncologist at the time of the original encounter, toxicities were retrospectively graded using the common terminology criteria for adverse events (CTCAE), version 5.0 (2017). Toxicities, which developed more than 30 days after treatment completion were categorized as late/chronic. One patient was excluded from chronic toxicity assessment due to inadequate follow-up.

\section{Statistical analysis}

When assessing descriptive variables, categorical variables were described using Pearson's chi-squared test or Fisher's exact test, as applicable. Continuous variables were described using Student's $t$-test or Wilcoxon rank-sum test. Multivariable regression analyses was performed to determine the independent association of clinical outcomes while adjusting for confounding variables. Logistic regression analyses was performed to determine any predictors of binary outcomes while adjust- ing for confounding variables. Cox proportional hazards analysis and Kaplan-Meier curves were used to assess time-to-event data.

\section{Results}

\section{Patient and tumor characteristics}

Clinical and pathologic characteristics for the 18 patients included in this study are summarized in Table 1. The majority of patients $(n=12,66.7 \%)$ had a primary disease of the distal vagina or vulva, and had FIGO stage II disease $(n=8,66.7 \%)$. Six patients $(33.3 \%)$ presented with secondary or recurrent cancer, most commonly endometrial in origin. The most common location for primary malignancy was the vulva, representing $66.7 \%(n=8 / 12)$ of patients with primary disease. In contrast, only $50 \%$ $(n=3 / 6)$ of those presenting with disease recurrence were classified as vulvar. Of all patients treated for vulvar disease, 7 were located either close to the urethra or at the vaginal introitus, 2 originated in the Bartholin gland, 1 involved the posterior fourchette and perianal area, and 1 patient had recurrent disease of the vulva treated 
with prior EBRT. With all patients considered, the most common histology was squamous cell carcinoma. Three patients $(16.7 \%)$ in total, all of whom had secondary/ recurrent disease, had received prior pelvic radiation. Two patients $(11.1 \%)$, both with primary disease, had undergone lymph node dissection prior to radiation. Both patients were found to have lymph node involvement at the time of surgery. The remaining 4 patients with lymph node involvement were diagnosed radiographically.

\section{Treatment planning and delivery}

Table 2 portrays radiation treatment details. All the patients completed EBRT, with a median dose of $45 \mathrm{~Gy}$ in 25 fractions, most frequently delivered through intensity modulated radiation therapy (IMRT) or volumetric modulated arc therapy (VMAT). This was followed by IGBT. The majority of patients $(n=15,83.3 \%)$ had MRI guidance for treatment planning, while the remaining patients $(n=3,16.7 \%)$ had CT guidance alone. The patients were

Table 1. Patient and disease characteristics, $n=18$

\begin{tabular}{|c|c|c|}
\hline Variable & Median & $\begin{array}{l}\text { Interquartile } \\
\text { range }\end{array}$ \\
\hline Age (years) & 62.0 & $55.4-67.6$ \\
\hline Weight (kg) & 83.6 & 74.1-94.3 \\
\hline \multirow[t]{2}{*}{$\mathrm{BMI}\left(\mathrm{kg} / \mathrm{m}^{2}\right)$} & 30.9 & $27.4-36.2$ \\
\hline & $n$ & $\%$ \\
\hline \multicolumn{3}{|l|}{ Locations of all patients } \\
\hline Distal vaginal & 7 & 38.9 \\
\hline Vulvar & 11 & 61.1 \\
\hline $\begin{array}{l}\text { Location of those with primary } \\
\text { malignancy }\end{array}$ & 12 & 66.7 \\
\hline Distal vaginal & 4 & 33.3 \\
\hline Vulvar & 8 & 66.7 \\
\hline Recurrent malignancy type & 6 & 33.3 \\
\hline Endometrial & 5 & 83.3 \\
\hline Vulvar & 1 & 16.7 \\
\hline \multicolumn{3}{|c|}{$\begin{array}{l}\text { FIGO stage for patients with primary } \\
\text { malignancy }\end{array}$} \\
\hline II & 8 & 66.7 \\
\hline III & 3 & 25.0 \\
\hline IV & 1 & 8.3 \\
\hline \multicolumn{3}{|l|}{ Histology } \\
\hline Squamous cell & 12 & 66.7 \\
\hline Endometrioid & 5 & 27.8 \\
\hline Poorly differentiated & 1 & 5.6 \\
\hline \multicolumn{3}{|l|}{ Lymph node involvement } \\
\hline Yes & 6 & 33.3 \\
\hline No & 12 & 66.7 \\
\hline \multicolumn{3}{|l|}{ Smoking history } \\
\hline Yes & 9 & 50.0 \\
\hline No & 9 & 50.0 \\
\hline
\end{tabular}

treated with IGBT to a median dose of 20.50 Gy (range, 15.0-27.5 Gy) in 3.5 fractions (range, 3-5 fx.). The most common fractionation for IGBT was $21 \mathrm{~Gy}$ in 3 fractions $(n=3,16.7 \%)$. IGBT was delivered with a Syed applicator in 14 patients $(77.8 \%)$, a Capri applicator in 3 patients $(16.7 \%)$, and a custom multichannel cylinder applicator in 1 patient $(5.6 \%)$. Fourteen patients $(77.8 \%)$ had systemic therapy applied for their treatment, all concurrent with radiation.

\section{Dosimetry}

All patients had dosimetric data available. Median high-risk clinical target volume (HR-CTV) $\mathrm{D}_{90}$ was 73.7 Gy. Fourteen patients $(77.8 \%)$ had HR-CTV $D_{90}$ $>70 \mathrm{~Gy}$, and of those 4 had $\mathrm{D}_{90}>75 \mathrm{~Gy}$. The remaining 4 patients had HR-CTV $\mathrm{D}_{90}<70 \mathrm{~Gy}$, with a range of 53.4-69.6 Gy. The patient with HR-CTV $\mathrm{D}_{90}$ of 53.4 Gy was treated to a lower dose due to a history of prior radiation. There was no association between HR-CTV $\mathrm{D}_{90}$ and cancer control $(\mathrm{HR}=1.023, p=0.81)$. The median $\mathrm{EQD}_{2}$ for the bladder, rectum, and urethra were $47.8 \mathrm{~Gy}, 52.3 \mathrm{~Gy}$, and $69.8 \mathrm{~Gy}$, respectively. All the patients met $\mathrm{EQD}_{2}$ constraints for the bladder, rectum, and urethra. $\mathrm{EQD}_{2}$ for the contoured OARs was not associated with any acute or chronic toxicity.

\section{Acute and chronic toxicities}

Overall rates of acute and chronic toxicities associated with the treatment were low and were predominately vulvar-, vaginal-, or skin-related. Grade 3 (G3) or higher acute toxicities included 1 (5.6\%) G3 vaginal stenosis, 6 (33.3\%) G3 dermatitis/mucositis, 2 (11.1\%) G3 vaginal pain, and 1 (5.6\%) G3 vaginal/vulvar infection. Grade 3 or higher late toxicities included $3(17.7 \%)$ G3 vaginal pain and $1(5.9 \%)$ G3 skin/vaginal necrosis. One instance of G3 vaginal pain resolved over time. The instance of G3 skin/ vaginal necrosis resolved after the surgery for recurrent disease. There were no instances of vaginal perforation, fistula formation, dyspareunia, urethral stricture, or G4 or G5 toxicities reported. All the patients with HR-CTV $D_{90}$

Table 2. Radiation treatment details

\begin{tabular}{lcc} 
Radiation treatment & $n$ & $\%$ \\
\hline \begin{tabular}{l} 
External beam radiation therapy (EBRT) details \\
\cline { 2 - 3 } Dose (cGy)
\end{tabular} & \\
\hline \begin{tabular}{lcc}
\hline 4500 & 2 & 11.1 \\
\hline 4500 & 12 & 66.7 \\
\hline$>4500$ & 4 & 22.2 \\
\hline EBRT type & & \\
\hline IMRT/VMAT & 17 & 94.4 \\
\hline 3D conformal & 1 & 5.6 \\
\hline Brachytherapy treatment details & & \\
\hline Planning type & & \\
\hline CT & 3 & 16.7 \\
\hline MRI & 15 & 83.3
\end{tabular}
\end{tabular}


Table 3. Follow-up and recurrence

\begin{tabular}{|c|c|c|c|c|}
\hline \multicolumn{5}{|l|}{ Follow-up (months) } \\
\hline Mean (SD) & Median & IQR & Min. & Max. \\
\hline 20.6 & 15.61 & $4.2-39.0$ & 0.93 & 54 \\
\hline Recurrence & & & $n$ & $\%$ \\
\hline Total number of recurrences & & & 5 & 27.8 \\
\hline Local recurrence & & & 3 & 16.7 \\
\hline Distant recurrence & & & 1 & 5.6 \\
\hline Simultaneous regional and di & t recurrenc & & 1 & 5.6 \\
\hline
\end{tabular}

$>75$ Gy had at least $1 \mathrm{G} 3$ toxicity. One patient suffered from G3 acute dermatitis/mucositis, chronic vaginal pain, and chronic vaginal/skin necrosis. One patient had G3 acute vaginal pain and acute dermatitis/mucositis. One patient suffered from G3 acute dermatitis/mucositis alone, and one patient had G3 acute vaginal stenosis alone.

\section{Follow-up, recurrence, and survival}

Follow-up and recurrence data are described in Table 3. At a median follow-up of 15.6 months, there were a total of 5 recurrences. Of these recurrences, $3(16.7 \%)$ were local recurrences alone, $1(5.6 \%)$ was a distant recurrence alone, and $1(5.6 \%)$ was a simultaneous regional and distant recurrence. All 3 local recurrences were in patients who originally presented with primary vulvar disease. Of these, two were periurethral or at the vaginal introitus, and one was in a patient with a posterior fourchette/perianal tumor; however, in the latter case, the recurrence was outside of the brachytherapy volume. Two had CT-guided brachytherapy (BT) alone. One patient had a lower HR-CTV $\mathrm{D}_{90}$ of $69.6 \mathrm{~Gy}$. One- and two-year progression-free survival were $93.8 \%$ and $75.0 \%$, respectively. Figure 3 depicts overall survival. One- and twoyear overall survival were $83.9 \%$ and $73.4 \%$, respectively. Stepwise selection test for association with recurrence of any type and overall survival was unremarkable.

\section{Discussion}

Radiation plays a critical role in the treatment of vaginal and vulvar cancer, either as definitive treatment or as part of a multimodal approach to management $[7,13,15$ 18]. A recent retrospective study by Yang et al. including 124 patients with vaginal cancers of varying stages was conducted to analyze clinical characteristics and survival of patients with primary vaginal cancers treated with various modalities, and demonstrated no difference in progression-free survival or disease-specific survival for patients treated with primary radiation compared to primary surgery for stage I-IVA disease [13]. It is important to note that given the proximity of the vagina to the bladder, rectum, and urethra, surgical intervention is often associated with significant morbidity $[19,20]$. As such, although the study by Yang et al. reported similar outcomes with the use of primary surgery or radiation, definitive radiation, either with or without chemotherapy, is often pursued in an attempt to avoid the toxicity associated with surgery. Within radiation treatments available, the inclusion of BT has been associated with improved outcomes in several studies [15, 21-23].

Several studies have been performed on the use of IGBT in vaginal cancers with promising results $[8,11,24-$ 26]. A study by Manuel et al. examining the use of IGBT compared to BT without image-guidance for the treatment of vaginal cancer, showed improved 2-year local control $(93 \%$ vs. $71 \%, p=0.03)$ and disease-free interval (86\% vs. $54 \%, p=0.04)$ with IGBT. This study also demonstrated a statistically significant decrease in G3 genitourinary toxicity ( $0 \%$ vs. $22 \%, p=0.002)$ in IGBT group compared to patients treated without IGBT [11]. A separate study by Beriwal et al. evaluating clinical outcomes of thirty patients with vaginal cancer, treated with image-guided interstitial brachytherapy showed 1- and 2-year locoregional control of $84.4 \%$ and $78.8 \%$, respectively, with no G3 or higher gastrointestinal complications, and only 2 patients with G3 vaginal ulceration and G4 vaginal necrosis [3]. Another study by Dimopoulos et al. investigating the feasibility of MRI-guided BT for 13 patients with locally advanced vaginal cancer, demonstrated 3-year local control of $92 \%$ with 2 patients developing a fistula, 1 case of periurethral necrosis, and 1 patient suffering from vaginal obliteration [16].

Although studies on the use of IGBT for the treatment of vaginal cancer have been performed, most have low numbers of patients with distal vaginal cancers, and no study has focused on the treatment of distal vaginal cancers alone. It is possible that this is in part because distal cancers are scarce, as vaginal cancers most commonly arise in the proximal one third of this organ [27]. For example, the cohort of patients with distal vaginal cancers included in the Manuel study represented only $19.4 \%$ of

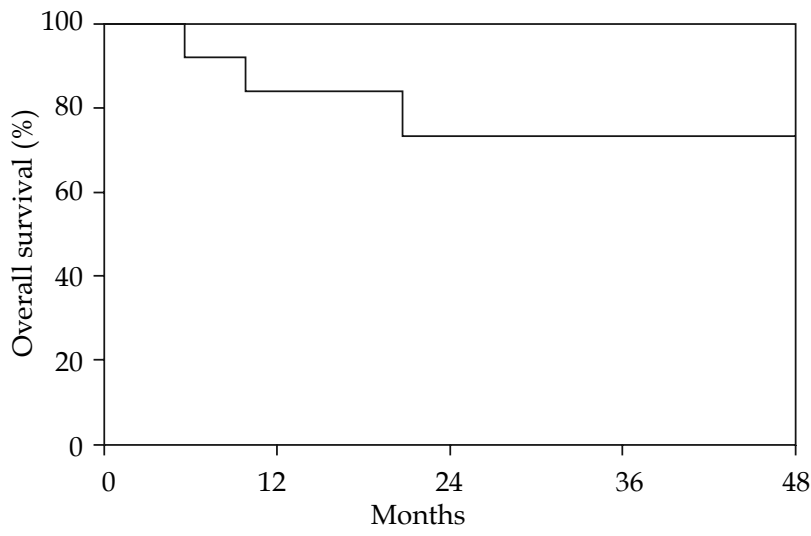

Fig. 3. Overall survival $(n=18)$ 
the entire patient population [11]. Only $11 \%$ of patients in a separate study by Lee et al. examining the clinical outcomes of 44 women with vaginal recurrence of endometrial cancer treated with IGBT had distal vaginal tumors [25]. The relative lack of distal vaginal cancers included in these studies is of particular importance because it is thought that the distal vagina is more sensitive to radiation compared to the more proximal vagina and, therefore, could be at higher risk for toxicity with brachytherapy [28]. This is evident in a recent retrospective review by Goodman et al., in which $33.9 \%$ of the included 67 patients had tumors located in the lower vagina alone. This study demonstrated a $10.4 \%$ rate of G3/4 genitourinary and gastrointestinal toxicity, including four cases of vaginal fistula formation and four instances of vaginal necrosis. At a median follow-up of 2.68 years, $11.9 \%$ of patients $(n=8)$ experienced local recurrence, either in isolation or in combination with both regional or distant recurrence, similar to the current study [29].

While few studies exist on the use of IGBT for vaginal cancers, even fewer exist on the treatment of vulvar malignancies, likely because surgery is currently the standard of care for early-stage disease, with primary radiation traditionally reserved for those deemed medically inoperable or unresectable, or those with recurrent disease [30]. The studies that do exist evaluating the use of IGBT for vulvar cancers have demonstrated mixed results. One single-institution experience, including 14 women with locally advanced or recurrent vulvar cancer, treated with high-dose-rate (HDR)-BT with or without EBRT, showed that while arrest of cancer growth or tumor regression was achieved in all patients, 8 patients (57.1\%) experienced a relapse. One-year progression-free survival was only $33 \%$. In this retrospective study, only two patients (14.3\%) experienced G3 toxicity [8]. In contrast to the high-rate of recurrence in this study, an experience by Mahantshetty et al. including 38 patients, treated with HDR-BT as a part of definitive radiation (76.3\%), adjuvant $(15.8 \%)$, or salvage $(7.9 \%)$ therapy, demonstrated a 5-year local control of $77 \%$ [31]. A separate study by Thibault et al. on the use of HDR-BT for Bartholin's gland carcinoma included 5 patients total, three treated with adjuvant radiation and two treated with definitive chemoradiation. Similar to the current study, all patients received EBRT. While the Thibault's study demonstrated $100 \%$ local control at a median follow-up of 78 months, $60 \%$ of patients suffered G3 or greater toxicity [32].

The current study is the first to focus on the clinical outcomes associated with the use of IGBT for the treatment of distal vaginal and vulvar cancers. The majority of the patients included in the study had primary vulvar cancer that were treated with definitive chemoradiation due to tumor location, for which surgery was likely to result in excess morbidity due to proximity to the urethra and anus. The patients with vulvar cancer were treated with brachytherapy, as opposed to dose-escalated EBRT, because it was assumed to be able to spare OARs, particularly the urethra, better than higher dose EBRT based on location as described above. This study demonstrated clinically meaningful local control, with low rates of both acute and late genitourinary and gastrointestinal toxicity, with only $16.7 \%$ of patients experiencing local recurrence at a median follow-up of 15.61 months. All local recurrences were observed in patients who presented with primary vulvar cancers. It is important to note that, given the rare nature of vulvar cancer itself, there is currently a paucity of data on the use of BT in the treatment of vulvar cancers. As such, no standardized guidelines exist for the optimal utilization of BT in the treatment of this disease. The relatively high rates of local recurrence for vulvar cancers treated with IGBT in both the current study and the study by Kellas-Ślęczka, calls into question the need for dose escalation in the treatment of vulvar cancer; although this would almost certainly increase toxicity associated with treatment, even in the era of IGBT, given the relatively high-rate of G3 skin and vaginal toxicity observed in the current study.

This study was limited by a small number of patients treated at a single-institution, with a relatively low number of events, thus limiting its power. A second limitation was the heterogeneity of BT dosing utilized, which could be difficult to apply to a wide array of patients. A final limitation was its retrospective nature, with toxicities graded based on physician description of toxicity, if not explicitly graded by the treating radiation oncologist at the time of treatment or follow-up. However, given the rarity of distal vaginal and vulvar malignancies, it is imperative that there are data demonstrating the efficacy and safety of radiotherapy for their treatment. This patients' population would likely benefit from a prospective trial to further address these questions and potentially maximize treatment-related outcomes.

\section{Conclusions}

In summary, the results of this study indicate that women with distal vaginal and vulvar cancers, either primary or recurrent in nature, can be treated with highdose-rate interstitial brachytherapy and external beam radiation, achieving meaningful cancer control.

\section{Disclosure}

The authors report no conflict of interest.

\section{References}

1. Glaser SM, Beriwal S. Brachytherapy for malignancies of the vagina in the 3D era. J Contemp Brachytherapy 2015; 7: 312-318.

2. Vargo JA, Kim H, Houser CJ et al. Image-based multichannel vaginal cylinder brachytherapy for vaginal cancer. Brachytherapy 2015; 14: 9-15.

3. Beriwal S, Rwigema J-CM, Higgins E et al. Three-dimensional image-based high-dose-rate interstitial brachytherapy for vaginal cancer. Brachytherapy 2012; 11: 176-180.

4. Carter JS, Downs LS. Vulvar and vaginal cancer. Obstet Gynecol Clin North Am 2012; 39: 213-231.

5. Beriwal S, Demanes DJ, Erickson B et al. American Brachytherapy Society consensus guidelines for interstitial brachytherapy for vaginal cancer. Brachytherapy 2012; 11: 68-75.

6. Huertas A, Dumas I, Escande A et al. Image-guided adaptive brachytherapy in primary vaginal cancers: A monocentric experience. Brachytherapy 2018; 17: 571-579. 
7. De Ieso PB, Mullassery V, Shrimali R et al. Image-guided vulvovaginal interstitial brachytherapy in the treatment of primary and recurrent gynecological malignancies. Brachytherapy 2012; 11: 306-310.

8. Kellas-Ślęczka S, Białas B, Fijałkowski M et al. Interstitial high-dose-rate brachytherapy in locally advanced and recurrent vulvar cancer. J Contemp Brachytherapy 2016; 8: 32-40.

9. Gebhardt BJ, Vargo JA, Kim H et al. Image-based multichannel vaginal cylinder brachytherapy for the definitive treatment of gynecologic malignancies in the vagina. Gynecol Oncol 2018; 150: 293-299.

10. Rijkmans EC, Nout RA, Rutten IHHM et al. Improved survival of patients with cervical cancer treated with image-guided brachytherapy compared with conventional brachytherapy. Gynecol Oncol 2014; 135: 231-238.

11. Manuel MM, Cho LP, Catalano PJ et al. Outcomes with image-based interstitial brachytherapy for vaginal cancer. Radiother Oncol 2016; 120: 486-492.

12. Westerveld H, Nesvacil N, Fokdal L et al. Definitive radiotherapy with image-guided adaptive brachytherapy for primary vaginal cancer. Lancet Oncol 2020; 21: e157-e167.

13. Yang J, Delara R, Magrina J et al. Management and outcomes of primary vaginal cancer. Gynecol Oncol 2020; 159: 456-463.

14. Rajagopalan MS, Kannan N, Kim H et al. Urethral dosimetry and toxicity with high-dose-rate interstitial brachytherapy for vaginal cancer. Brachytherapy 2013; 12: 248-253.

15. Leung E, D'Souza D, Bachand F et al. MRI-based interstitial brachytherapy for vaginal tumors: A multi-institutional study on practice patterns, contouring, and consensus definitions of target volumes. Brachytherapy 2019; 18: 598-605.

16. Dimopoulos JCA, Schmid MP, Fidarova E et al. Treatment of locally advanced vaginal cancer with radiochemotherapy and magnetic resonance image-guided adaptive brachytherapy: dose-volume parameters and first clinical results. Int J Radiat Oncol 2012; 82: 1880-1888.

17. Stock RG, Chen ASJ, Seski J. A 30-year experience in the management of primary carcinoma of the vagina: analysis of prognostic factors and treatment modalities. Gynecol Oncol 1995; 56: 45-52.

18. National Comprehensive Cancer Network. Vulvar Cancer (Squamous Cell Carcinoma) NCCN Evidence Blocks. https:/ / www.nccn.org/professionals/physician_gls/pdf/vulvar_ blocks.pdf

19. Maggioni A, Roviglione G, Landoni F et al. Pelvic exenteration: Ten-year experience at the European Institute of Oncology in Milan. Gynecol Oncol 2009; 114: 64-68.

20. Matsuo K, Mandelbaum RS, Adams CL et al. Performance and outcome of pelvic exenteration for gynecologic malignancies: A population-based study. Gynecol Oncol 2019; 153: 368-375.

21. Rajagopalan MS, Xu KM, Lin JF et al. Adoption and impact of concurrent chemoradiation therapy for vaginal cancer: A National Cancer Data Base (NCDB) study. Gynecol Oncol 2014; 135: 495-502.

22. Reshko LB, Gaskins JT, Metzinger DS et al. The impact of brachytherapy boost and radiotherapy treatment duration on survival in patients with vaginal cancer treated with definitive chemoradiation. Brachytherapy 2021; 20: 75-84.

23. Orton A, Boothe D, Williams N et al. Brachytherapy improves survival in primary vaginal cancer. Gynecol Oncol 2016; 141: 501-506.

24. Castelnau-Marchand P, Escande A, Mazeron R et al. Brachytherapy as part of the conservative treatment for primary and recurrent vulvar carcinoma. Brachytherapy 2017; 16: 518-525.

25. Lee LJ, Damato AL, Viswanathan AN. Clinical outcomes following 3D image-guided brachytherapy for vaginal recurrence of endometrial cancer. Gynecol Oncol 2013; 131: 586-592.
26. Lee LJ, Damato AL, Viswanathan AN. Clinical outcomes of high-dose-rate interstitial gynecologic brachytherapy using real-time CT guidance. Brachytherapy 2013; 12: 303-310.

27. Rajaram S, Maheshwari A, Srivastava A. Staging for vaginal cancer. Best Pract Res Clin Obstet Gynaecol 2015; 29: 822-832.

28. Hintz BL, Kagan AR, Chan P et al. Radiation tolerance of the vaginal mucosa. Int J Radiat Oncol 1980; 6: 711-716.

29. Goodman CD, Mendez LC, Velker V et al. 3D image-guided interstitial brachytherapy for primary vaginal cancer: A multi-institutional experience. Gynecol Oncol 2021; 160: 134-139.

30. Koh WJ, Greer BE, Abu-Rustum NR et al. Vulvar Cancer, Version 1.2017, NCCN Clinical Practice Guidelines in Oncology. J Natl Compr Canc Netw 2017; 15: 92-120.

31. Mahantshetty U, Naga P, Engineer R et al. Clinical outcome of high-dose-rate interstitial brachytherapy in vulvar cancer: A single institutional experience. Brachytherapy 2017; 16: 153160 .

32. Thibault I, Lavallée MC, Aubin S et al. Management of Bartholin's gland carcinoma using high-dose-rate interstitial brachytherapy boost. Brachytherapy 2013; 12: 500-507. 